\title{
A method for chronological intravital imaging of bovine oocytes during in vitro maturation
}

Petersen, Morten Møller; Hansen, Michael; Avery, Birthe Margit; Bøgh, Ingrid Brück

\section{Published in:}

Microscopy and Microanalysis

DOI:

$10.1017 / \mathrm{S} 1431927608080872$

Publication date:

2008

Document version

Publisher's PDF, also known as Version of record

Citation for published version (APA):

Petersen, M. M., Hansen, M., Avery, B. M., \& Bøgh, I. B. (2008). A method for chronological intravital imaging of bovine oocytes during in vitro maturation. Microscopy and Microanalysis, 14(6), 549-560.

https://doi.org/10.1017/S1431927608080872 


\title{
A Method for Chronological Intravital Imaging of Bovine Oocytes during In Vitro Maturation
}

\author{
Morten R. Petersen, ${ }^{1, \star}$ Michael Hansen, ${ }^{2}$ Birthe Avery, ${ }^{1}$ and Ingrid B. Bøgh ${ }^{1}$ \\ ${ }^{1}$ Department of Large Animal Sciences, Section for Veterinary Reproduction and Obstetrics, Faculty of Life Sciences, \\ Copenhagen University, Dyrlaegevej 68, 1870 Frederiksberg C, Copenhagen, Denmark \\ ${ }^{2}$ Department of Plant Biology, Faculty of Life Sciences, Copenhagen University, Thorvaldsensvej 40, 1870 Frederiksberg C, \\ Copenhagen, Denmark
}

\begin{abstract}
Oocyte maturation is known to affect the chances for successful fertilization, embryonic development, establishment of pregnancy and delivery of a live, healthy, and viable offspring. Two-photon laser scanning microscopy (TPLSM) has previously been used to evaluate early embryonic development without a detectable impairment of subsequent development, but has never been applied to assess mammalian oocytes throughout in vitro maturation (IVM). Visualization of structures within live oocytes during IVM, followed by fertilization and embryo culture, may improve the understanding of oocyte maturation. To visualize structures within bovine oocytes using TPLSM, it is necessary to remove the cumulus cells that normally surround the oocyte during maturation. Repeated visualization of structures within the same oocyte is possible, if movement of the oocyte can be avoided. In this article, we describe the development of a method for repeated intravital imaging of denuded bovine oocytes using an upright TPLSM equipped with a specially constructed incubator. Oocytes were stained with Hoechst 33258, and the nuclear structures were evaluated. Oocyte fertilization rate was not affected by TPLSM exposure, but the developmental capacity of the denuded oocytes was significantly reduced. This is, to our knowledge, the first article describing repeated intravital imaging during mammalian oocyte maturation using TPLSM.
\end{abstract}

Key words: bovine, oocyte maturation, two-photon laser scanning microscopy, live cell imaging, poly-L-lysine, Hoechst

\section{INTRODUCTION}

Within the last 25 years, our knowledge on the cellular and molecular processes in gametes around fertilization and early embryos has vastly increased, not the least as a result of the advancement of assisted reproductive techniques. In vitro maturation (IVM), in vitro fertilization (IVF), and subsequent culture of the fertilized oocyte to the blastocyst stage can now be achieved in several species, although research has predominantly focused on the human, murine, and bovine species.

Several studies emphasize the importance of an optimal oocyte maturation to increase the chances of fertilization, embryonic development, establishment of pregnancy, and delivery of a live, healthy, and viable offspring (Krisher, 2004; Sirard et al., 2006). Therefore, much attention is currently placed on studying oocyte maturation.

Received July 10, 2008; accepted July 21, 2008

${ }^{\star}$ Corresponding author. E-mail: mmpe@life.ku.dk

\section{Intravital Imaging}

Squirrell and coworkers have shown that two-photon laser scanning microscopy (TPLSM) can be used for intravital imaging without detectable impairment of subsequent development of the visualized zygote or embryo (Squirrell et al., 1999; Squirrell \& White, 2004). With the increasing availability of highly specific vital fluorophores, this technique allows repeated visualization of several morphological structures or molecular processes throughout early development, enabling a direct link between these events and the developmental capacity of the individual oocyte or embryo. Being able to directly correlate the visualized features with the developmental capacity of the oocyte or embryo minimizes the number of specimens necessary to examine, allowing research on scarcely available material and reduce costs. With regard to visualization of embryos, TPLSM is superior in obtaining images from deep within embryos compared to other visualization techniques, e.g., confocal microscopy (Centonze \& White, 1998).

An essential requirement for successful and trustworthy data generation through intravital imaging is to 
control temperature, humidity, and atmosphere composition on the microscope stage while visualizing the subjects of interest. Because oocyte maturation is affected by culture conditions (Lonergan et al., 2003), optimal and constant culture conditions are fundamental.

\section{Oocyte Maturation}

During oocyte maturation, several structural and functional changes occur in the nucleus and cytoplasm that eventually supports fertilization and early embryonic development: e.g., in the nucleus, chromatin configuration proceeds from prophase I to metaphase II, and in the cytoplasm polyadenylation of mRNA changes as well as the localization of RNA, mitochondria, and cortical granules (Hyttel et al., 1997; Brevini Gandolfi \& Gandolfi, 2001).

Close interaction and communication between the oocyte and the surrounding somatic cumulus cells is essential during oocyte maturation to support the cytoplasmatic maturation, but appears unnecessary for nuclear maturation to take place (Hashimoto et al., 1998; Mori et al., 2000; Eppig, 2001; Chang et al., 2005).

\section{Intravital Imaging of Oocyte Maturation}

Considering the importance of oocyte maturation described above and the advantages of intravital imaging, we decided to develop a TPLSM time-lapse setup that would allow repeated intravital observations during oocyte maturation. To establish and evaluate the visualization platform, it was decided to evaluate the nuclear changes during maturation by using the DNA-specific fluorophore Hoechst 33258. Visualization of the chromatin changes during meiosis was chosen because basic key points concerning the morphology and kinetic of the different meiotic stages has previously been established in bovine oocytes and are described in detail in the literature (Willadsen, 1973; Motlik et al., 1978; Hyttel et al., 1997). Furthermore, the bovine IVF methodology is well established and grants a stable system with more than $85 \%$ of the oocytes progressing through meiosis I and II (Avery et al., 2003). Thus, the nucleus was considered an excellent "model-structure" for the establishment and validation of the visualization platform.

Before data should be collected, the visualization platform should meet several requirements: (1) it must allow visualization of structures within the maturing oocyte, (2) oocyte movement must be avoided to allow repeated evaluations of the same structures, and (3) developmental capacity of the oocytes should be maintained.

\section{Partial or Complete Denudation of Cumulus Oophorus Complexes}

Visualization of structures within the oocyte is not possible without removing at least part of the cumulus cells, but as mentioned, close cumulus-oocyte contact is indispensable to support normal oocyte maturation. Partial denudation, in which a cumulus-oophorus-complex (COC), is aspirated into a glaspipette, with a diameter slightly smaller than the oocyte, is one approach to "create a window" in the cumulus cell layers to allow visualization without compromising developmental capacity (Fig. 1) (Petersen et al., 2005).

During IVM, the cumulus layer surrounding the oocyte expands causing subtle changes in oocyte positioning, which renders repeated evaluations of the same structures within a specific oocyte difficult. Attaching the partially denuded COCs to the bottom of the petridish with poly-L-lysine (PLL) is one approach to avoid movement of the COCs without compromising the developmental capacity (Petersen et al., 2005). Even though partial denudation seemed a feasible approach to allow visualization of structures within the oocyte, preliminary trials showed that nuclear chromatin could only be visualized in 1 out of 50 examined partially denuded oocytes, which was less than acceptable.

Another approach to allow visualization of structures within the oocyte is to remove all the cumulus cells before oocyte maturation. To support IVM of totally denuded oocytes, cumulus cell suspensions or cumulus cell conditioned media have previously been used; nevertheless developmental capacity was still reduced compared to intact in vitro matured COCs (Hashimoto et al., 1998; Geshi et al., 2000; Tanghe et al., 2003). Luciano et al. (2005) reported recently that blastocyst rates from oocytes denuded before IVM were similar to those originating from intact COCs, if the denuded oocytes were matured in close vicinity to intact COCs. IVM of denuded oocytes in coculture with intact COCs therefore was considered a feasible way to support nuclear as well as cytoplasmatic maturation in the present study.

\section{Objectives}

The present article describes the establishment of a method for chronological intravital imaging of bovine oocytes on an upright two-photon microscope during IVM. The objectives were to (1) develop an incubation system that allows in vitro oocyte maturation and can be used in connection with the available TPLSM, (2) establish a method to avoid movement of denuded oocytes using PLL, while maintaining developmental capacity in a coculture setup (experiment 1 ), (3) validate whether the developmental capacity of the oocytes is maintained under these culture conditions and visualize nuclear chromatin within the maturing denuded oocyte (experiment 2).

\section{Materials and Methods}

All the plastic was purchased from Nunc (VWR International, Albertslund, Denmark), and all reagents and media were from Sigma-Aldrich (Vallensbæk, Denmark), unless otherwise indicated. The media were prepared with freshly produced Milli-Q water (Millipore, Hedehusene, Denmark). 


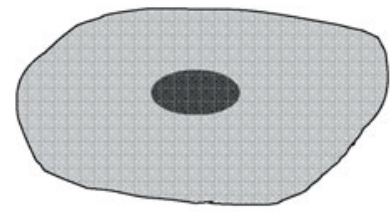

A

Intact cumulus oophorus complex

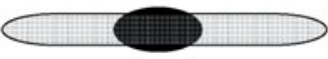

B

Partially denuded

cumulus oophorus complex

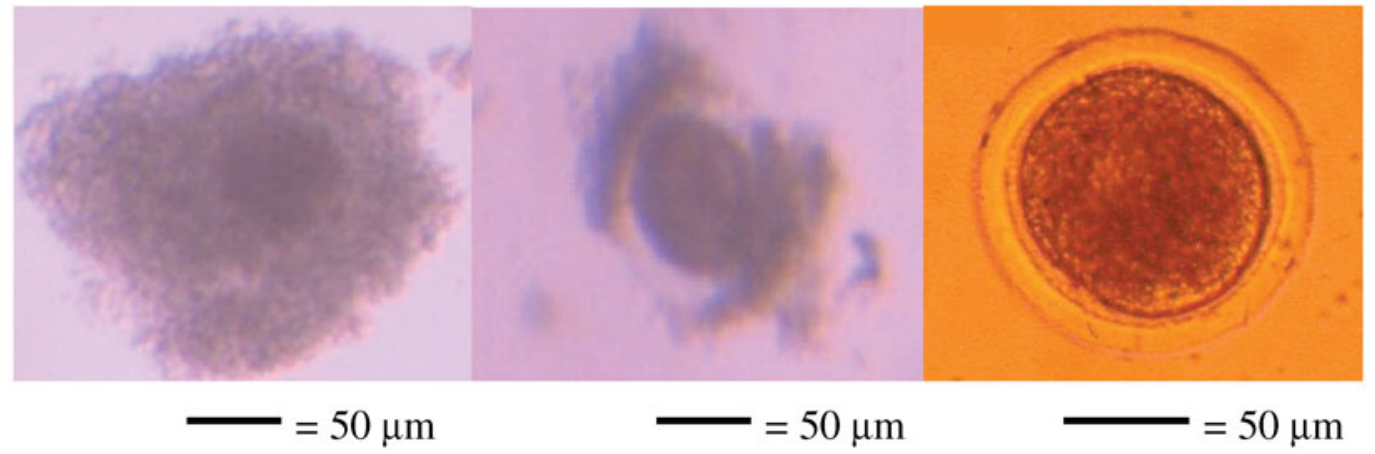

Figure 1. Schematic illustration and picture of (A) an intact cumulus oophorus complex, (B) a partially denuded cumulus complex, and (C) a denuded oocyte.

Bovine embryos were produced according to standard procedures as previously described (Parrish et al., 1986; Holm et al., 1999; Avery \& Greve, 2000; Avery et al., 2003; Vejlsted et al., 2005). Modifications to these procedures are described in detail in the following sections.

\section{Oocyte Retrieval and Handling}

Ovaries were collected from Danish cows and heifers at a local slaughterhouse and transported within $1 \mathrm{~h}$ to the IVF laboratory in thermo boxes submerged in sterile $0.9 \%$ saline $\left(30-33^{\circ} \mathrm{C}\right)$. Antral follicles with surface diameters of 3 to $15 \mathrm{~mm}$ were evacuated using a vacuum pump connected with an 18-gauge short beveled needle into a $50-\mathrm{mL}$ tube, which contained $1 \mathrm{~mL}$ Hepes buffered wash medium (TCM199, M 2520) supplemented with $20 \mathrm{U} / \mathrm{mL}$ heparin to prevent clotting of the follicular fluid. After aspiration and retrieval, the COCs were washed three times in TCM-199 without heparin. After washing, $40 \%$ of the oocytes were denuded by vortexing for $2 \mathrm{~min}$ in TCM-199. Before IVM, all oocytes (intact COCs and denuded) were washed once in IVM medium.

\section{Developmental Capacity of Denuded Oocytes and COCs Matured in Coculture on PLL or Not (Experiment 1)}

\section{Treatment Groups}

Oocytes were distributed randomly to three different treatment groups for IVM: standard protocol with intact
COCs matured without PLL (group I), intact COCs (group IIa) and denuded oocytes (group IIIa) combined in coculture in a 1:1 ratio, intact COCs (group IIb) and denuded oocytes (group IIIb) combined in coculture in a $1: 1$ ratio in a petri dish coated with PLL $\left(5 \mu \mathrm{L} / \mathrm{cm}^{2}\right.$, $0.1 \mathrm{mg} / \mathrm{mL}$, Sigma P-4832).

\section{In Vitro Maturation, Fertilization, and Culture}

Oocytes were in vitro matured in groups of 30-40 oocytes in $60-\mathrm{mm}$ dishes in a volume of $13 \mu \mathrm{L}$ IVM medium/COC with mineral oil overlay ( $\mathrm{M}$ 8410) in an atmosphere of $5 \% \mathrm{CO}_{2}$ in humidified air at $38.5^{\circ} \mathrm{C}$. The IVM medium was Dulbecco's Modified Eagle Medium (D 5523), supplemented with $50 \mathrm{ng} / \mathrm{mL}$ epidermal growth factor (E 4127), $2 \mathrm{U} / \mathrm{mL}$ Suigonan Vet. (Intervet Denmark A/S, Skovlunde, Denmark), $50 \mu \mathrm{g} / \mathrm{mL}$ gentamycin (G 1264), 5\% estrous cow serum (ECS; Danish Institute for Food and Veterinary Research, Copenhagen, Denmark), and $10 \mu \mathrm{g} / \mathrm{ml}$ of Hoechst 33258 (Invitrogen ${ }^{\circledR}, \mathrm{H}-1398,2630$ Taastrup, Denmark). After $24 \mathrm{~h}$ of IVM (22.5 to $25 \mathrm{~h}$ ), the oocytes (COCs and denuded oocytes) were washed once in TALP medium and then placed in $0.5 \mathrm{~mL}$ of TALP medium without oil overlay in four-well dishes. The TALP medium contained $6 \mathrm{mg} / \mathrm{mL}$ BSA (A 4919), $30 \mu \mathrm{g} / \mathrm{mL}$ heparin (H 3149), $0.25 \mathrm{mM}$ sodium pyruvate ( $\mathrm{P}$ 3662), $20 \mu \mathrm{M}$ penicillamine (P 4875), $10 \mu \mathrm{M}$ hypotaurine (H 1384), and $1 \mu \mathrm{M}$ epinephrine (E 4250). The COCs and denuded oocytes were then coincubated for $24 \mathrm{~h}$ with frozen-thawed washed semen from one fertile bull (Vikinggenetics ${ }^{\circledR}$, 
Denmark) at a final sperm concentration of $2.5 \times 10^{6} / \mathrm{mL}$ in $5 \% \mathrm{CO}_{2}$ in humidified air at $38.5^{\circ} \mathrm{C}$. After IVF, intact COCs and denuded oocytes were separated and kept separate for whole mount fixation or in vitro culture. Cumulus cells were removed from intact COCs by vortex agitation for $1 \mathrm{~min}$. For each of the four replicates, 5-12 inseminated oocytes were whole mount fixated and later stained with aceto orcein (Avery \& Greve, 2000) to determine fertilization status. After washing, groups of 20 to 25 inseminated oocytes were transferred to $0.1-\mathrm{mL}$ droplets covered with mineral oil in SOFaac medium with $10 \%$ ECS and cultured until day 8 after insemination in a modular Incubator Chamber (patent no. 5352414; BillupsRothenberg, Inc. Del Mar, CA, USA) at $38.5^{\circ} \mathrm{C}$ in $5 \% \mathrm{CO}_{2}$, $5 \% \mathrm{O}_{2}, 90 \% \mathrm{~N}_{2}$.

\section{Determination of Fertilization Status and Blastocyst Evaluation}

Presumptive zygotes were whole mount fixated and stained with aceto orcein (Avery \& Greve, 2000) for assessment of fertilization status according to the number of pronuclei (PN) as follows: 0 PN (not fertilized); 1 PN (activation of the oocyte without fertilization); 2 PN (normal fertilization); >2 PN (polyspermic fertilization).

On day 8 after IVF, the blastocyst rates were recorded based on the number of inseminated oocytes (presumptive zygotes), and the developmental stage (kinetics) and morphology of the blastocysts were assessed. Assuming that morphology as well as kinetics follow a normal distribution, and that different normal distributions cannot share more than two values, it was possible to calculate a normal distribution for each of the treatment groups and thus determine the average kinetics and morphology score for each of the five treatment groups (group I, IIa, IIb, IIIa, and IIIb). Each blastocyst was assigned a kinetic score between 1 and 3: score 1 (hatched blastocyst $(\mathrm{H})$ ), score 2 (expanded blastocyst (XB)), and score 3 (nonexpanded blastocyst (BL)). This scoring system describes the developmental stages that are identified on day 8; the nonexpanded blastocyst (containing only a small blastocoel volume), the expanded blastocyst (increasing blastocoel volume), and the hatched blastocyst, the most developed stage, which no longer is surrounded by the zona pellucida. Likewise, the morphology score was assessed: score 1 (excellent morphology with a compact and distinct inner cell mass as well as regular morphology of the trophectodermal cells); score 2 (good morphology with a small or less distinct inner cell mass and/or few degenerated trophectodermal cells/slight fragmentation); score 3 (poor morphology with diffuse or no inner cell mass, several fragmented or degenerated trophectodermal cells, or developmental arrest). After kinetic and morphologic scoring, an average score was calculated for each treatment group.

\section{Intravital Imaging during Oocyte Maturation (Experiment 2)}

\section{Microscope Incubator-Heating Device and Incubation Chamber}

Initially, a small commercially available incubation chamber was tested, but overnight temperature measurements showed large fluctuations. As an alternative, a special incubator was constructed of foamed PVC sheets $(10 \mathrm{~mm})$ surrounding the microscope stage and the objective revolver (Fig. 2). Two double-layered plastic pipes (inner diameter $10 \mathrm{~cm}$ ) was attached to each side of the incubator, creating a circle with the heating device to maintain the desired temperature. A PT100 temperature sensor, placed in the incubator and connected to the heating device, controlled the temperature $\left(38.5^{\circ} \mathrm{C}\right)$ by activating the heating element when the temperature was to low. Temperature fluctuations were prevented by a continuous flow of air through "the circle," independent on whether the heating element was active or not. A constant temperature was reached after $5 \mathrm{~h}$ of equilibration. To obtain an atmosphere that would support oocyte maturation, an almost airtight incubation chamber was constructed of 2-mm Plexiglas (Fig. 3). The incubation chamber, covered with a lid, was placed directly on the microscope stage. The "opening" in the microscope stage floor was adjusted with a special made metal ring to fit a $90-\mathrm{mm}$ petri dish. A $60-\mathrm{mm}$ petri dish containing the IVM media, and oocytes were then placed into the $90-\mathrm{mm}$ petri dish. A circular opening in the incubation chamber lid $(4 \mathrm{~cm}$ in diameter) allowed entering and movement of the water immersion lens. To close the opening, a Plexiglas "objective collar" was placed on top of the lid. The "objective collar" had a tight fit around the middle part of the water immersion lens during image acquisition (Fig. 3). Thus, it was possible to freely move the water immersion lens and at the same time maintain an almost airtight incubation chamber. The incubation chamber was directly linked to a gasholder providing a constant flow of premade mixed gas with a constant flow of $10 \mathrm{~nL} / \mathrm{h}$ of $5 \% \mathrm{CO}_{2}$ in air. Oocyte maturation was performed under oil, as in the IVF laboratory, which controlled the osmolarity of the IVM media by preventing evaporation. Using this setup, temperature, atmosphere composition, and humidity were controlled.

\section{Staining Protocol and Microscope Settings}

Staining of the DNA of denuded oocytes and COCs was performed by adding $10 \mu \mathrm{g} / \mathrm{mL}$ Hoechst 33258 to the IVM medium. Pilot experiments documented that visualization of oocyte chromatin could be conducted following a 15min incubation in Hoechst 33258 IVM medium (data not shown). Visualization was performed no earlier than $30 \mathrm{~min}$ after transfer of oocytes into Hoechst 33258 IVM medium.

Imaging was performed on a TPLSM (Leica TCS SP2/ MP; Leica Microsystems, Bensheim, Germany) equipped with a two-photon laser (Mai Tai, 780-920 nm, Spectra- 


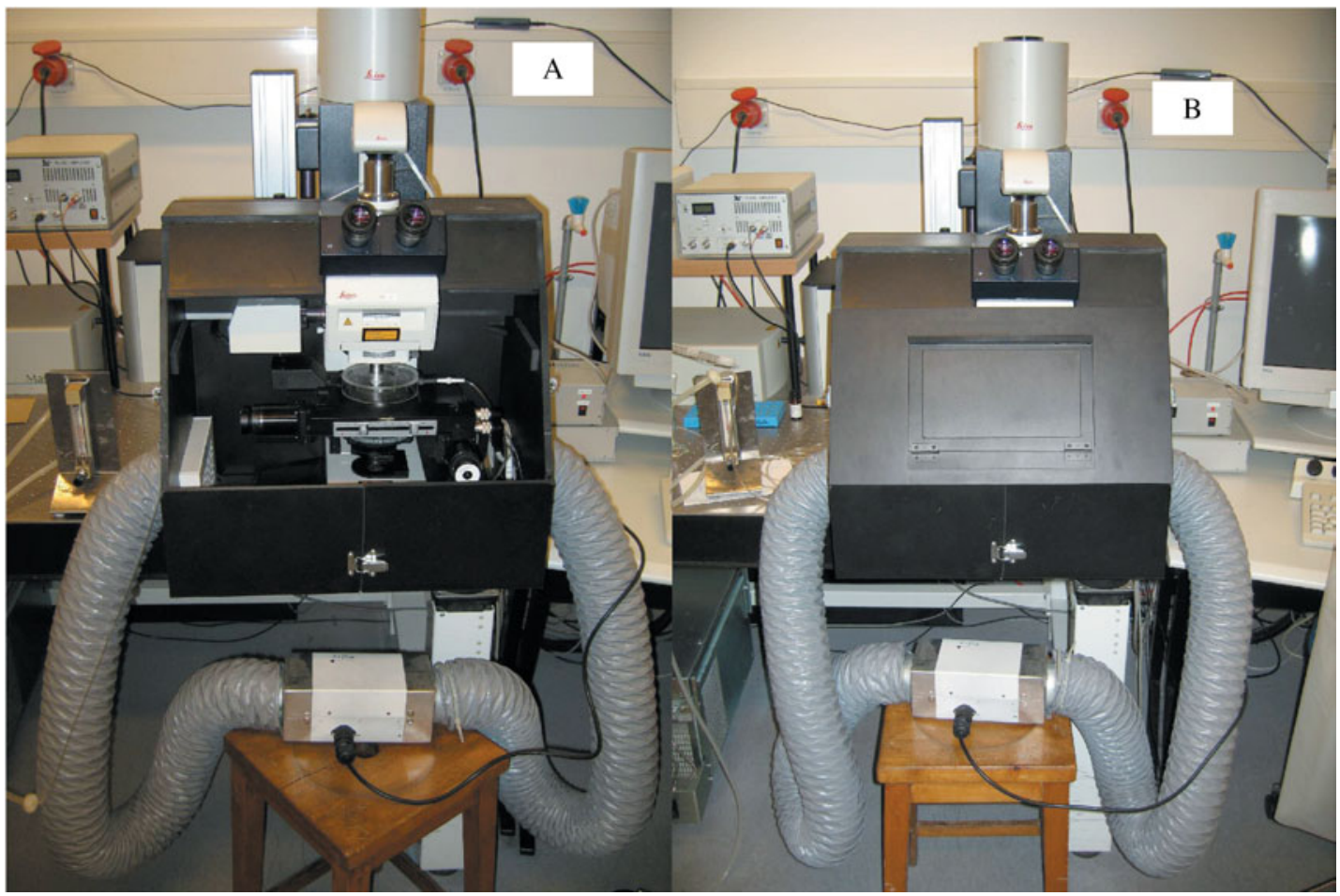

Figure 2. "Microscope incubator." A: Front part of the microscope is surrounded by the microscope incubator (black box). Top part of the microscope incubator is removed to allow visualization of incubation chamber including gas inlet. Air is moved clockwise through the tubing using a continuous flow of air from the heating device (placed on the small chair). B: As A, but with front part attached and access door closed.

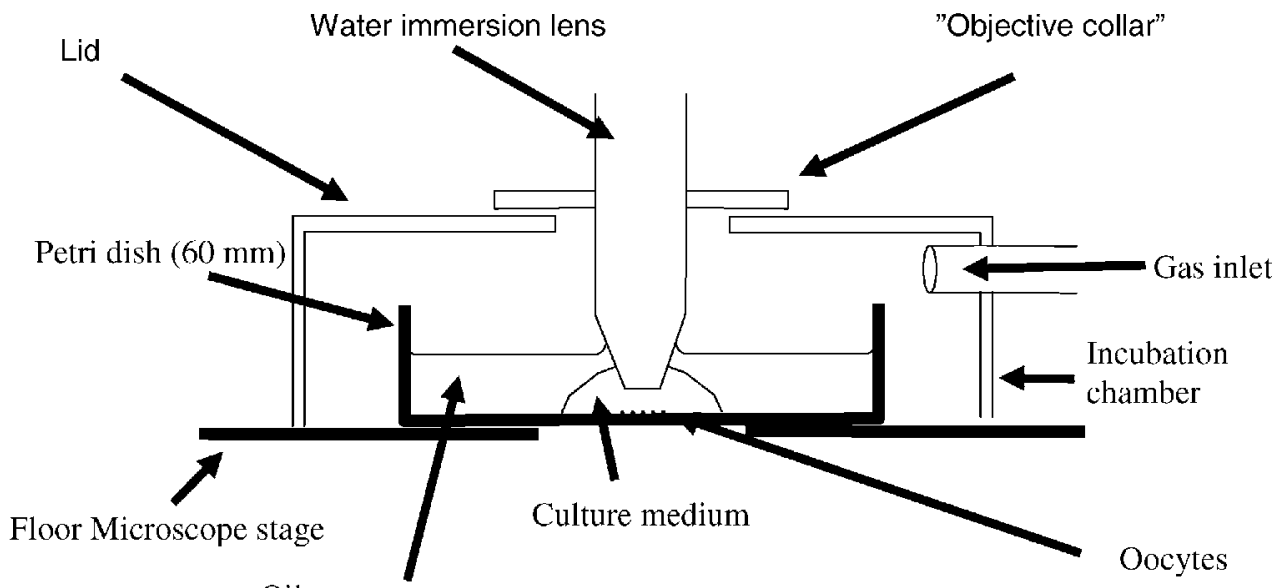

Oil cover

Figure 3. Schematic drawing of the incubation chamber. See text for detailed description of the different components. Note that the $90-\mathrm{mm}$ petri dish is not shown.

Physics, Mountain View, CA, USA). The microscope was equipped with a motorized stage, controlled by the microscope software. With the software, specific positions were defined ( $x, y$ and $z$ coordinates), which allowed return to the same position several times and conduct repeated evaluations of the same oocyte. For imaging the laser was set to
$780 \mathrm{~nm}$ to excite Hoechst 33258. Photons were detected using a nondescan detector placed immediately after the objective to maximize the signal. No emission filter was used. The objective was a Leica Microsystems HCX APO Lx63/0.90W, placed directly into the maturation media ("water immersion lens"). 
$10 \mathrm{~mm}$

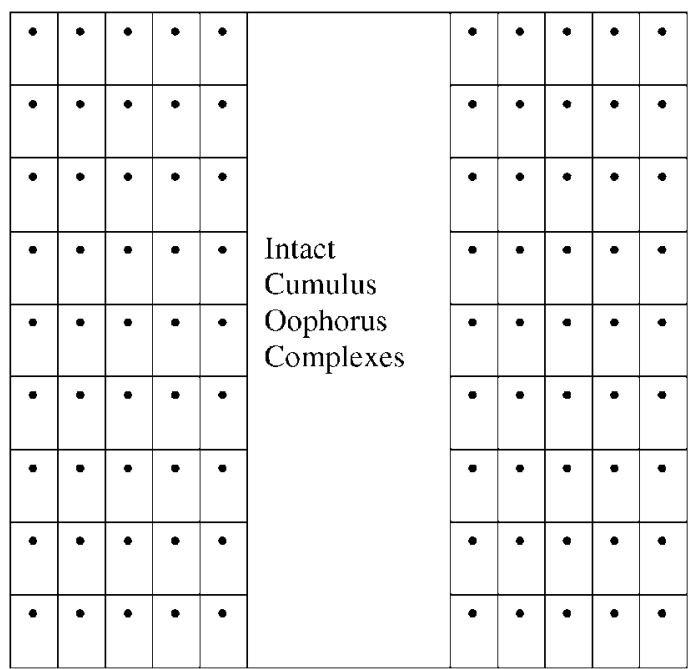

$10 \mathrm{~mm}$

Figure 4. Schematic illustration of the quadratic pattern $(10 \times$ $10 \mathrm{~mm}$ ) scratched on the outside of the bottom of a $60-\mathrm{mm}$ petri dish used for intravital imaging. Intact COCs were placed in the central third of the dish with completely denuded oocytes $(\bullet)$ placed on each side.

For each visualized oocyte, a stack of images was obtained. The stack encompassed the entire oocyte in three dimensions. The format of the stack $(512 \times 512$ pixels, 8 bits) was in the horizontal direction $238.2 \times 238.2 \mu \mathrm{m}$, and in the vertical direction $120 \mu \mathrm{m}$ with $2 \mu \mathrm{m}$ between sections. During image acquisition, each line was scanned only once $(1.6 \mathrm{~s})$ to reduce phototoxicity and photobleaching. Each oocyte was exposed to the laser for a total of $480 \mathrm{~s}$. The top and bottom position of each visualized oocyte was manually defined before every evaluation. After collecting a stack of images from each of the evaluated oocytes, data were saved on an external hard drive to avoid a software crash before evaluation was performed again. Image visualization and three-dimensional analysis were performed with the software of the TCS SP2. Each oocyte was evaluated five times during maturation $(22.5 \mathrm{~h})$.

\section{Oocyte Maturation Performed in the "Microscope Incubator"}

Before maturation, denuded oocytes as well as COCs were placed in a $60-\mathrm{mm}$ petri dish with PLL (Fig. 4) and moved very gentle to the TPLSM. To ease identification of each individual oocyte, one denuded oocyte was placed in each square (Fig. 4). The "markings" illustrated in Fig. 4 were "hand-scratched" on the bottom of the petri dish the day before use with the tip of a needle. During imaging, only denuded oocytes placed on one side of the intact COCs were evaluated/exposed, while denuded oocytes on the other side served as control/nonexposed (Fig. 4). Oocyte handling and medium used for IVM were performed as described for experiment 1 . After IVM and evaluation, denuded oocytes were separated in evaluated and control oocytes. The evaluated oocytes were fertilized together with half of the intact COCs and the control oocytes were fertilized with the other half of intact COCs. After fertilization, oocytes were separated in denuded and intact COCs, and the cumulus cells of the intact COCs were removed using vortexing. On the day after fertilization, presumptive zygotes were either evaluated using whole mount fixation (two replicates), as described above, to determine fertilization rate or cultured for a total of 8 days to assess their capacity to develop into blastocysts (one replicate). Blastocyst kinetics and morphology were evaluated as described for experiment 1.

\section{Statistics}

Sample means and standard deviations (mean $\pm S D$ ) were calculated per replicate for the proportion of oocytes that were fertilized normally and developed into blastocysts. The effects of the different treatments were analyzed by Fisher's exact test by pairwise comparisons using a more stringent level of significance when more than two groups were compared to avoid errors of multiple interference or by using larger contingency tables and chi-square tests. The software program GraphPad InStat was used for the statistical calculations (Version 3.00 for Windows NT, GraphPad, San Diego, CA).

\section{Results}

\section{Developmental Capacity of Denuded Oocytes and COCs Matured in Coculture on PLL or Not (Experiment 1)}

There were no significant differences $(p>0.05)$ in $2 \mathrm{PN}$ rates between standard IVM group $(33 / 42=79 \%)$ and the intact COCs matured in the presence of denuded oocytes with or without a supportive layer of PLL $(16 / 25=70 \%$ and $32 / 46=64 \%$, Table 1 ). The fertilization rates of denuded oocytes with or without a supportive layer of PLL $(12 / 22=56 \%$ and $27 / 48=54 \%)$ were not significantly different from intact COCs. There was, however, a significant linear trend $(p<0.022)$ between the standard IVM (group I), the intact COCs in coculture with denuded oocytes (groups IIa and IIIa), and the denuded oocytes (groups IIb and IIIb), showing that denuded oocytes had the lowest fertilizing capacity of the three groups (Table 1).

Of the oocytes matured using the standard IVM protocol (COCs without coculture) 23\% (26/112) developed into blastocysts, which is not significantly different from intact COCs matured in the coculture system, but significantly different from oocytes denuded before maturation in coculture (no PLL $(5 / 123=4 \%)$, on PLL $(4 / 127=3 \%), p<$ 0.0001 , Table 2 ). The capacity of intact COCs matured in coculture to develop into a blastocyst was similar, irrespectively whether maturation proceeded on PLL or not. How- 
Table 1. Pronuclear Rate $24 \mathrm{~h}$ after IVF of Intact COCs Matured Using Our Standard Protocol and Intact COCs and Denuded Oocytes (DOs) Matured in Coculture with or without PLL.

\begin{tabular}{lccccc}
\hline & $\begin{array}{c}\text { Zero } \\
\text { pronuclei } \%\end{array}$ & $\begin{array}{c}\text { One } \\
\text { pronuclei } \% \\
(n)\end{array}$ & $\begin{array}{c}\text { Two } \\
\text { pronuclei } \% \\
(n)\end{array}$ & $\begin{array}{c}>\text { Two } \\
\text { pronuclei } \% \\
(n)\end{array}$ & $\begin{array}{c}\text { Total number } \\
\text { of oocytes }\end{array}$ \\
Treatment group & $19 \pm 4$ & 2 & $79 \pm 7$ & - & 42 \\
Group I (Standard IVM) & $(8 / 42)$ & $(1 / 42)$ & $(33 / 42)$ & $(0 / 42)$ & 25 \\
Group IIa (COCs, -PLL) & $20 \pm 11$ & 12 & $64 \pm 16$ & 4 & 46 \\
Group IIIa (COCs, +PLL) & $(5 / 25)$ & $(3 / 25)$ & $(16 / 25)$ & $(1 / 25)$ & 4 \\
Group IIb (DOs, - PLL) & $22 \pm 19$ & 4 & $70 \pm 16$ & $(2 / 46)$ & 22 \\
Group IIIb (DOs, +PLL) & $(10 / 46)$ & $(2 / 46)$ & $54 \pm 19$ & $(0 / 22)$ & 2 \\
& $(86 \pm 18$ & 9 & $(12 / 22)$ & $(1 / 48)$ & 48 \\
\end{tabular}

Note: Results expressed as mean $\pm S D$. Based on four replicates. $5 \times 2$ Chi-square used. Significant negative linear trend in fertilization rates from group 1 (standard IVM), IIa + IIb (intact COCs), and IIIa + IIIb (DOs).

Table 2. Blastocyst Rate, Kinetic and Morphology Scores for Intact COCs In Vitro Matured under Standard IVM Conditions or Intact COCs and Denuded Oocytes (DOs) In Vitro Matured in Coculture with or without PLL.

\begin{tabular}{|c|c|c|c|c|c|c|c|c|c|}
\hline \multirow[b]{3}{*}{ Treatment groups } & \multirow[b]{3}{*}{$\begin{array}{c}\% \text { D8 BL } \\
(n)\end{array}$} & \multicolumn{4}{|c|}{ Kinetic score } & \multicolumn{4}{|c|}{ Morphology score } \\
\hline & & 1 & 2 & 3 & & 1 & 2 & 3 & \\
\hline & & $\begin{array}{c}\% \mathrm{H} \\
(n)\end{array}$ & $\begin{array}{c}\% \mathrm{XB} \\
(n)\end{array}$ & $\begin{array}{c}\% \mathrm{BL} \\
(n)\end{array}$ & Mean & $\begin{array}{l}\% \mathrm{~A} \\
(n)\end{array}$ & $\begin{array}{c}\% \mathrm{~B} \\
(n)\end{array}$ & $\begin{array}{c}\% \mathrm{C} \\
(n)\end{array}$ & Mean \\
\hline Group I (Standard IVM) & $\begin{array}{l}23 \pm 1^{a} \\
(26 / 112)\end{array}$ & $\begin{array}{c}19 \\
(5 / 26)\end{array}$ & $\begin{array}{c}50 \\
(13 / 26)\end{array}$ & $\begin{array}{c}31 \\
(8 / 26)\end{array}$ & 2.1 & $\begin{array}{c}52 \\
(14 / 26)\end{array}$ & $\begin{array}{c}27 \\
(7 / 26)\end{array}$ & $\begin{array}{c}19 \\
(5 / 26)\end{array}$ & 1.7 \\
\hline Group IIa (COCs, - PLL) & $\begin{array}{l}17 \pm 2^{\mathrm{a}} \\
(24 / 138)\end{array}$ & $\begin{array}{c}4 \\
(1 / 24)\end{array}$ & $\begin{array}{c}63 \\
(15 / 24)\end{array}$ & $\begin{array}{c}33 \\
(8 / 24)\end{array}$ & 2.3 & $\begin{array}{c}38 \\
(9 / 24)\end{array}$ & $\begin{array}{c}46 \\
(11 / 24)\end{array}$ & $\begin{array}{c}17 \\
(4 / 24)\end{array}$ & 1.8 \\
\hline Group IIIa (COCs, + PLL) & $\begin{array}{l}21 \pm 11^{\mathrm{a}} \\
(32 / 154)\end{array}$ & - & $\begin{array}{c}63 \\
(20 / 32)\end{array}$ & $\begin{array}{c}37 \\
(12 / 32)\end{array}$ & 2.4 & $\begin{array}{c}47 \\
(15 / 32)\end{array}$ & $\begin{array}{c}38 \\
(12 / 32)\end{array}$ & $\begin{array}{c}16 \\
(5 / 32)\end{array}$ & 1.7 \\
\hline Group IIb (DOs, -PLL) & $\begin{array}{l}4 \pm 5^{b} \\
(5 / 123)\end{array}$ & $\overline{(0 / 5)}$ & $\begin{array}{c}80 \\
(4 / 5)\end{array}$ & $\begin{array}{c}20 \\
(1 / 5)\end{array}$ & 2.6 & $\begin{array}{c}40 \\
(2 / 5)\end{array}$ & $\begin{array}{c}20 \\
(1 / 5)\end{array}$ & $\begin{array}{c}40 \\
(2 / 5)\end{array}$ & 1.8 \\
\hline Group IIIb (DOs, + PLL) & $\begin{array}{l}3 \pm 4^{b} \\
(4 / 127)\end{array}$ & $\overline{(0 / 4)}$ & $\begin{array}{c}75 \\
(3 / 4)\end{array}$ & $\begin{array}{c}25 \\
(1 / 4)\end{array}$ & 2.3 & $\begin{array}{c}50 \\
(2 / 4)\end{array}$ & $\overline{(0)}$ & $\begin{array}{c}50 \\
(2 / 4)\end{array}$ & 2.0 \\
\hline
\end{tabular}

Note: Results expressed as mean $\pm S D$. Based on four replicates. Data analyzed by Fisher's exact test, rows with different superscript are significantly different $p<0.0001$. D8 BL $=$ day 8 blastocyst. Kinetic score: $\mathrm{H}$, hatched blastocyst; XB, expanded blastocyst; BL, nonexpanded blastocyst. Morphology score 1-3: excellent, good, and poor (see text for further details on kinetic and morphologic scores).

ever, there was a strong reduction in the blastocyst rate, when oocytes were denuded before maturation $(p<0.0001)$ (Table 2). Blastocyst formation was not different between denuded oocytes matured on PLL or not. The blastocysts in the different treatment groups reached similar stages of development and had similar mean morphology scores (Table 2). However, only few fertilized oocytes developed into blastocysts, when denuded before maturation.

\section{Intravital Imaging during Oocyte Maturation (Experiment 2)}

\section{Visualization of Chromatin}

A total of 49 denuded oocytes were visualized during the three replicates. Oocyte chromatin was visualized in 10/49 within the initial $7 \mathrm{~h}$ following IVM start, while in the 

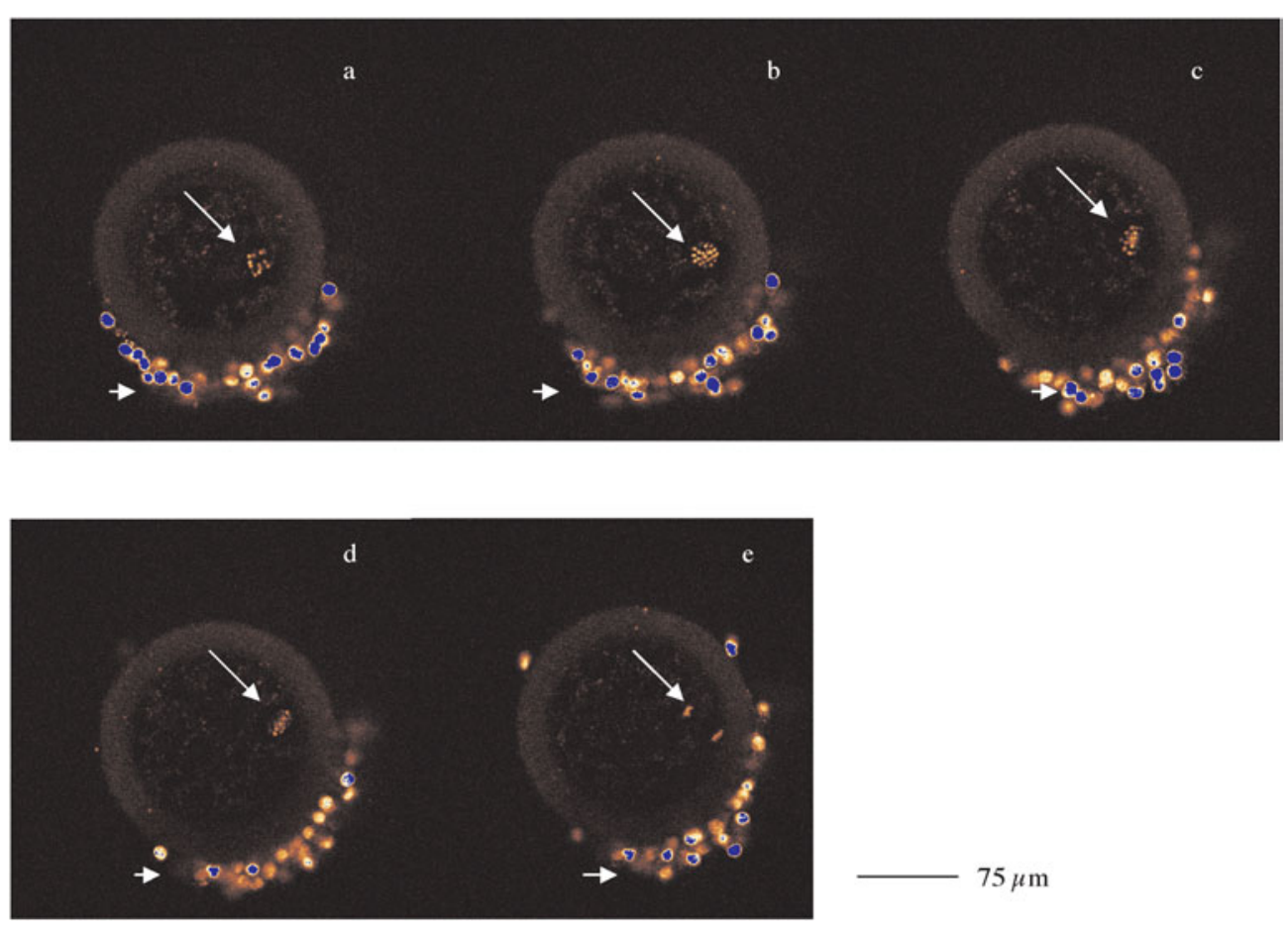

Figure 5. Chronological images of the same bovine oocyte with a few cumulus cells on one pole labeled with Hoechst 33258 visualized at difference time point: (A) 9 h, (B) 12 h, (C) 16 h, (D) 17 h, and (E) 21 h after IVM start. Long white arrows indicate chromatin configuration at the different time points. The images represent selected sections, where most chromatin was visible, explaining why the same cumulus cells (short white arrows) are not visualized in each section. Because the top and bottom of each oocyte was redefined manually between each visualization round, subtle changes in chromatin position could not be determined. (A) Nine hours after initiation of in vitro maturation, the nuclear chromatin is starting to condense. After condensation, the chromatin aligns in metaphase I at (B) $12 \mathrm{~h},(\mathbf{C}) 16 \mathrm{~h}$, and (D) $17 \mathrm{~h}$ after IVM start. After reaching metaphase, the chromatin separates (anaphase and telophase). (E) The oocyte has reached telophase I ( $21 \mathrm{~h}$ after IVM start). Signal intensity is illustrated by different colors: yellow $=$ low intensity, blue $=$ medium intensity, white = high intensity. Note the strong signal from the nucleus of the few cumulus cells attached to the oocyte (lower part of the oocyte) compared to the oocyte chromatin. Scale bar $=35 \mu \mathrm{m}$.

period from 12-15 h until the end of IVM chromatin could be visualized in $40 / 49=82 \%$ of the evaluated oocytes (Fig. 5). Only meiotic stages before, at, or beyond early MI stage were visualized. In all of the visualized oocytes, excitation from Hoechst-stained mitochondrial DNA could be registered. DNA in extruded polar bodies or cumulus cells attached to the zona pellucida were also visualized, when located within $80 \mu \mathrm{m}$ of the part of the oocyte closest to the objective.

\section{Effects of Intravital Imaging during Oocyte Maturation on Developmental Capacity}

The described visualization platform allowed detailed studies on the chromatin configuration of the same oocyte over time (Fig. 5). To determine the influence of repeated TPLSM exposure on oocyte health, half of the denuded oocytes in the "microscope incubator" were not exposed to multipho- ton laser scanning serving as control for the evaluated group. There were no significant differences in the capacity between intact COCs $(43 / 60=72 \%)$, denuded $(15 / 32=$ $47 \%)$, or denuded and evaluated oocytes $(13 / 23=57 \%)$ to undergo normal fertilization (Table $3, p<0.056$ ). It should be noted that the $p$-value were close to the level of significance $(p<0.05)$.

The effect of multiphoton laser exposure on oocyte developmental capacity was assessed after fertilization of evaluated denuded, as well as nonevaluated denuded oocytes and intact COCs. The blastocyst rate for intact COCs matured in coculture in the "microscope incubator" was $32 \%(23 / 73)$. Of the 26 oocytes evaluated during maturation, 2 oocytes developed into blastocysts. None of the nonevaluated denuded oocytes developed into blastocysts (Table 4). Only few of the oocytes that were denuded before maturation developed into blastocysts (Table 4), similar to the results from experiment 1 (Table 2). 
Table 3. Pronuclear Rate of Intact COCs, Denuded Oocytes, and Evaluated Denuded Oocytes Matured in the "Microscope Incubator."

\begin{tabular}{lccccc}
\hline & $\begin{array}{c}\text { Zero } \\
\text { pronuclei } \% \\
(n)\end{array}$ & $\begin{array}{c}\text { One } \\
\text { pronuclei } \% \\
(n)\end{array}$ & $\begin{array}{c}\text { Two } \\
\text { pronuclei } \% \\
(n)\end{array}$ & $\begin{array}{c}>\text { Two } \\
\text { pronuclei \% } \\
(n)\end{array}$ & $\begin{array}{c}\text { Total number } \\
\text { of oocytes }\end{array}$ \\
\hline Intact COCs & $18 \pm 2$ & 3 & $72 \pm 2$ & $8 \pm 7$ & 60 \\
& $(11 / 60)$ & $(2 / 60)$ & $(42 / 60)$ & $(5 / 60)$ & 32 \\
Denuded oocytes & $34 \pm 30$ & $15 \pm 7$ & $47 \pm 41$ & 3 & $(1 / 32)$ \\
Evaluated denuded oocytes & $(11 / 32)$ & $(5 / 32)$ & $(15 / 32)$ & - & 23 \\
& $35 \pm 31$ & 9 & $57 \pm 17$ & & \\
\hline
\end{tabular}

Note: Based on two replicates. $3 \times 2$ Chi-square test used.

Table 4. Blastocyst Rates of Intact COCs, Denuded Oocytes, and Evaluated Denuded Oocytes Matured in the "Microscope Incubator" and Fertilized and Cultured for 8 Days.

\begin{tabular}{|c|c|c|c|c|c|c|c|c|c|}
\hline \multirow[b]{3}{*}{ Treatment group } & \multirow[b]{3}{*}{$\begin{array}{c}\% \text { D8 BL } \\
(n)\end{array}$} & \multicolumn{4}{|c|}{ Kinetic score } & \multicolumn{4}{|c|}{ Morphology score } \\
\hline & & 1 & 2 & 3 & & 1 & 2 & 3 & \\
\hline & & $\begin{array}{c}\% \mathrm{H} \\
(n)\end{array}$ & $\begin{array}{c}\% \mathrm{XB} \\
(n)\end{array}$ & $\begin{array}{c}\% \mathrm{BL} \\
(n)\end{array}$ & Mean & $\begin{array}{c}\% \mathrm{~A} \\
(n)\end{array}$ & $\begin{array}{c}\% \mathrm{~B} \\
(n)\end{array}$ & $\begin{array}{c}\% \mathrm{C} \\
(n)\end{array}$ & Mean \\
\hline Coculture COCs on PLL & $\begin{array}{c}32 \\
(23 / 73)\end{array}$ & $\begin{array}{c}4 \\
(1 / 23)\end{array}$ & $\begin{array}{c}35 \\
(8 / 23)\end{array}$ & $\begin{array}{c}61 \\
(14 / 23)\end{array}$ & 2.6 & $\begin{array}{c}26 \\
(6 / 23)\end{array}$ & $\begin{array}{c}39 \\
(9 / 23)\end{array}$ & $\begin{array}{c}35 \\
(8 / 23)\end{array}$ & 2.1 \\
\hline Denuded oocyte on PLL & $\begin{array}{c}0 \\
(0 / 37)\end{array}$ & - & - & - & - & - & - & - & - \\
\hline $\begin{array}{l}\text { Evaluated denuded oocyte } \\
\text { on PLL }\end{array}$ & $\begin{array}{c}8 \\
(2 / 26)\end{array}$ & - & $\begin{array}{c}50 \\
(1 / 2)\end{array}$ & $\begin{array}{c}50 \\
(1 / 2)\end{array}$ & 2.5 & $\begin{array}{r}100 \\
(2 / 2)\end{array}$ & - & - & 1 \\
\hline
\end{tabular}

Note: Based on one replicate. D8 BL, day 8 blastocyst; kinetic score: H, hatched blastocyst; XB, expanded blastocyst; BL, nonexpanded blastocyst. Morphology score 1-3: excellent, good, and poor (see text for further details on kinetic and morphologic scores).

\section{Discussion}

\section{Developmental Capacity of Denuded Oocytes Matured in Coculture}

The data from the present study indicate that denudation of oocytes before maturation negatively affects developmental capacity, even though the denuded oocytes are placed next to intact COCs during maturation. In the IVM protocol described by Luciano et al. (2005), denuded oocytes and intact bovine COCs were matured in very close vicinity and had direct contact to each other. Compared to this, the denuded oocytes in our study were placed between 50 and $300 \mu \mathrm{m}$ apart from the COCs (Fig. 4). Potentially this increased distance is responsible for a lower concentration of the putative diffusible factors secreted from the intact COCs, as suggested by Luciano et al. (2005). Further studies combining (1) cumulus cell conditioned media, (2) cumulus cells in suspension, (3) increased ratio between intact
COCs and denuded oocytes, (4) denuded oocytes surrounded directly by COCs, and/or (5) a different volume of IVM medium used in the coculture setup might increase the developmental capacity of denuded oocytes to the level similar or close to intact COCs.

\section{Intravital Imaging during Oocyte Maturation}

The present article describes for the first time a method that permits chronological intravital imaging of chromatin during IVM of bovine oocytes using an upright TPLSM. Visualization of structures within the oocyte was made possible by total denudation of the COCs before IVM and oocyte movement was prevented using PLL. Unfortunately only meiotic stages before, at, or beyond early MI stage could be visualized, indicating that a certain level of chromatin condensation is necessary for visualization.

Using this setup, we could demonstrate that the fertilizing capacity of examined denuded oocytes was not signifi- 
cantly different from nonevaluated intact COCs, although the subsequent developmental capacity reflected by blastocyst rate was reduced. This indicates that TPLSM based intravital imaging can be performed during oocyte maturation. However, to obtain images from a high percentage of oocytes, denudation is a necessity, which by itself reduces developmental capacity. From the present data it is not possible to conclude whether reduced developmental capacity in the evaluated denuded oocytes is a consequence of phototoxicity or denudation.

Traditionally, oocyte morphology at the subcellular level is evaluated after fixation. Using these invasive techniques, it is possible to recover high resolution images, but to correlate morphologic characteristics with, for example, embryo development, a high number of specimens must be evaluated. An alternative is intravital imaging, which allows a direct link between visualized events and the subsequent capacity to form an embryo. To correlate events occurring during oocyte maturation with subsequent embryo development, each individual oocyte and resulting embryo must be identified throughout IVM, fertilization, and embryo culture. In vitro oocyte maturation and fertilization can be performed in individual droplets without a negative impact on further development, but embryo development is reduced if individual culture is performed (Doherty et al., 1997; Khurana \& Niemann, 2000; Gopichandran \& Leese, 2006). With the Well Of the Well (WOW) system, the negative effects of individual culture can be overcome (Vajta et al., 2000), and simultaneously identification of the fertilized oocyte during IVC can be maintained. Individual culture during IVC was not performed in the present study, but should be included in future research to take full advantage of the assets of intravital imaging.

\section{Visualization of Chromatin}

Visualization of chromatin during IVM using TPLSM was only possible in a small fraction of partially denuded COCs, but in the majority of completely denuded oocytes. This discrepancy is due to optical differences between completely denuded and partially denuded oocytes.

Living specimens in general are difficult to visualize due to light scattering and mismatch in refractive indexes between membranes and cytoplasmatic components. Scattering and differences in refractive indexes are especially a problem in live bovine oocytes, which are covered by cumulus cells and surrounded by the zona pellucida. The high content of lipid droplets in oocytes increases the micro inhomogeneities in the cytoplasm and thus the amount of light scattered. Compared to, for example, confocal based or other linear optical microscopy, TPLSM is less sensitive to scattering making high resolution imaging possible, especially in "optically difficult" specimens (de Grauw \& Frederix, 2002). The efficiency of two-photon absorption depends on the physical properties of the dye molecule and on the spatial and temporal distribution of the excitation light
(Zipfel et al., 2003), whereas the emitted signal is insensitive to scatter (Centonze \& White, 1998). Even though chromatin could be visualized in a majority of the completely denuded oocytes, the intensity of the recovered signal was markedly less than the signal from the labeled DNA in the cumulus cells of the intact COCs (Fig. 5), indicating that the excitation efficiency within the oocyte is much less than in the cumulus cells. Two-photon microscopy is based on the simultaneous arrival of two photons (within approximately $0.5 \mathrm{fs}$ ), which makes the fluorescence signal quadratic dependent on the excitation intensity in the focus point (Helmchen \& Denk, 2005). Lack of an adequate intensity of photons to induce two-photon excitation could be an explanation for not being able to visualize chromatin within the partially denuded oocytes.

Oocyte chromatin was not visualized at the GV stage, but only from the early MI stage to the MII stage. This is different from the study of Lodde et al. (2007), who visualized different GV stages in living bovine oocytes using an inverted standard fluorescence microscope. Our decision to use multiphoton laser scanning microscopy was based upon previous reports documenting a low, if any, negative impact on embryo development using this imaging setup (Squirrell et al., 1999, 2003; Squirrell \& White, 2004). In previous studies, using a standard fluorescence microscope to correlate oocyte GV morphology to blastocyst formation, a lower blastocyst rate was found in visualized bovine and murine oocytes compared to control oocytes (Zuccotti et al., 1998; Lodde et al., 2007). UV light excitation of bovine oocyte chromatin labeled with Hoechst induces severe damage of the oocyte; e.g., if a MII oocyte is exposed, an abnormal response to parthenogenetic activation is seen (Bradshaw et al., 1995). In our experiments, two-photon excitation of oocyte chromatin labeled with Hoechst did not confirm this abnormal response to activation/fertilization because the visualized oocytes progressed through meiosis and were fertilized at a similar rate as the control oocytes.

Hoechst 33342 is potentially a more optimal fluorophore than Hoechst 33258, used in the present study, because it is more lipophilic and therefore more membrane permeable. However, Hoechst 33258 was used because data from previous exposure studies determined that oocyte exposure to $10 \mu \mathrm{g} / \mathrm{mL}$ of Hoechst 33258 during IVM did not affect subsequent developmental capacity (data not shown).

The morphology of the chromatin in the different meiotic stages was identical to the descriptions in the literature determined using invasive fixation techniques (Xu et al., 1986), indicating that the setup described here is a useful tool to perform detailed evaluations of chromatin configurations throughout meiosis. We hope in the future to use the established visualization platform to study nuclear as well as cytoplasmatic maturation of equine oocytes. Equine oocytes are in general less investigated than bovine oocytes and more difficult and expensive to recover (Choi et al., 1993; Hinrichs, 2005). 


\section{ConcLusions}

In this article we describe the development of a method to perform chronological intravital imaging of bovine oocytes during IVM using an upright TPLSM. Establishing the method consisted of several pilot experiments as well as two full-scale experiments: (1) we established a microscope incubator system that supported oocyte maturation as well as a standard incubator, (2) oocyte quality was maintained, while movement was prevented, by adhering the oocytes to the bottom of the petri dish with PLL (experiment 1), (3) visualization of oocyte chromatin using TPLSM did not affect the capacity for the oocyte to be fertilized in a form 2 pronuclei, but oocyte denudation, necessary to allow visualization of structures within the oocyte, negatively affected blastocyst formation capacity (experiment 2).

This is to our knowledge the first report on intravital imaging of mammalian oocyte maturation using TPLSM. A modified coculture system may provide more optimal conditions to maximize developmental capacity of denuded oocytes. The special optical conditions within the oocyte, and especially in the partially denuded COCs, render intravital imaging without compromising developmental capacity difficult. Intravital imaging can find more potential application in evaluating fertilized oocytes or embryos, because these developmental stages are free of cumulus cells.

\section{ACKNOWLEDGMENTS}

This research was funded by the Danish Agricultural and Veterinary Research Council project no. 23-02-0133. The authors greatly acknowledge the excellent technical assistance of Annedorte Roed, Lis Meyer, and Flemming Frederiksen.

\section{RefERENCES}

Avery, B. \& Greve, T. (2000). Effects of ethanol and dimethylsulphoxide on nuclear and cytoplasmic maturation of bovine cumulus-oocyte complexes. Molec Reprod Dev 55, 438-455.

Avery, B., Strobech, L., Jacobsen, T., Bogh, I.B. \& Greve, T. (2003). In vitro maturation of bovine cumulus-oocyte complexes in undiluted follicular fluid: Effect on nuclear maturation, pronucleus formation and embryo development. Theriogenol 59, 987-999.

Bradshaw, J., Jung, T., Fulka, J. \& Moor, R.M. (1995). UV irradiation of chromosomal dna and its effect upon mpf and meiosis in mammalian oocytes. Molec Reprod Dev 41, 503-512.

Brevini Gandolfi, T.A.L. \& Gandolfi, F. (2001). The maternal legacy to the embryo: Cytoplasmic components and their effects on early development. Theriogenol 55, 1255-1276.
Centonze, V.E. \& White, J.G. (1998). Multiphoton excitation provides optical sections from deeper within scattering specimens than confocal imaging. Biophys J 75, 2015-2024.

Chang, H.C., Liu, H., Zhang, J., Grifo, J. \& Krey, L.C. (2005). Developmental incompetency of denuded mouse oocytes undergoing maturation in vitro is ooplasmic in nature and is associated with aberrant Oct-4 expression. Hum Reprod 20, 1958-1968.

Choi, Y.H., Hochi, S., Braun, J., SAto, K. \& Oguri, N. (1993). In vitro maturation of equine oocytes collected by follicle aspiration and by the slicing of ovaries. Theriogenol 40, 959-966.

de Grauw, C.J. \& Frederix, P.L.T.M.G.H.C. (2002). Aberrations and penetration in in-depth confocal and two-photon-excitation microscopy. In Confocal and Two-Photon Microscopy, Diaspro, A. (Ed.), pp. 153-169. New York: Wiley-Liss, Inc.

Doherty, E.M.O., Wade, M.G., Hill, J.L. \& Boland, M.P. (1997). Effects of culturing bovine oocytes either singly or in groups on development to blastocysts. Theriogenol 48, 161-169.

Eppig, J.J. (2001). Oocyte control of ovarian follicular development and function in mammals. Reprod 122, 829-838.

Geshi, M., Takenouchi, N., Yamauchi, N. \& Nagai, T. (2000). Effects of sodium pyruvate in nonserum maturation medium on maturation, fertilization, and subsequent development of bovine oocytes with or without cumulus cells. Biol Reprod 63, 1730-1734.

Gopichandran, N. \& Leese, H.J. (2006). The effect of paracrine/ autocrine interactions on the in vitro culture of bovine preimplantation embryos. J Reprod Fertil 131, 269-277.

Hashimoto, S., Saeki, K., Nagao, Y., Minami, N., Yamada, M. \& Utsumi, K. (1998). Effects of cumulus cell density during in vitro maturation on the developmental competence of bovine oocytes. Theriogenol 49, 1451-1463.

Helmchen, F. \& Denk, W. (2005). Deep tissue two-photon microscopy. Nat Meth 2, 932-940.

Hinrichs, K. (2005). Update on equine ICSI and cloning. Theriogenol 64, 535-541.

Holm, P., Воотн, P.J., Schmidt, M.H., Greve, T. \& Callesen, H. (1999). High bovine blastocyst development in a static in vitro production system using sofaa medium supplemented with sodium citrate and myo-inositol with or without serumproteins. Theriogenol 52, 683-700.

Hyttel, P., Fair, T., Callesen, H. \& Greve, T. (1997). Oocyte growth, capacitation and final maturation in cattle. Theriogenol 47, 23-32.

Khurana, N.K. \& Niemann, H. (2000). Effects of oocyte quality, oxygen tension, embryo density, cumulus cells and energy substrates on cleavage and morula/blastocyst formation of bovine embryos. Theriogenol 54, 741-756.

KRISHER, R.L. (2004). The effect of oocyte quality on development. J Anim Sci 82, E14-E23.

Lodde, V., Modina, S., Galbusera, C., Franciosi, F. \& Luciano, A.M. (2007). Large-scale chromatin remodeling in germinal vesicle bovine oocytes: Interplay with gap junction functionality and developmental competence. Molec Reprod Dev 74, 740-749.

Lonergan, P., Robert, C., Gutierrez-Adan, A., Fair, T. \& Boland, M. (2003). Oocyte and embryo quality: Effect of origin, culture conditions and gene expression patterns. Reprod Dom Anim 38, 259-267.

Luciano, A.M., Lodde, V., Beretta, M.S., Colleoni, S., Lauria, A. \& Modina, S. (2005). Developmental capability of denuded 
bovine oocyte in a co-culture system with intact cumulusoocyte complexes: Role of cumulus cells, cyclic adenosine 3',5'-monophosphate, and glutathione. Mol Reprod Dev 71, 389-397.

Mori, T., Amano, T. \& Shimizu, H. (2000). Roles of gap junctional communication of cumulus cells in cytoplasmic maturation of porcine oocytes cultured in vitro. Biol Reprod 62, 913-919.

Motlik, J., Koefoed-Johnsen, H.H. \& Fulka, J. (1978). Breakdown of the germinal vesicle in bovine oocytes cultivated in vitro. J Exp Zool 205, 377-383.

Parrish, J.J., Susko-Parrish, J.L., Leibfried-Rutledge, M.L., Critser, E.S., Eyestone, W.H. \& First, N.L. (1986). Bovine in vitro fertilization with frozen-thawed semen. Theriogenol 25 , 591-600.

Petersen, M.M, Avery, B., Greve, T. \& Bøgh, I.B. (2005). Comparison of two methods to avoid movement of bovine oocytes during in vitro maturation. Reprod Fertil Dev 17, 298.

Sirard, M.A., Richard, F., Blondin, P. \& Robert, C. (2006). Contribution of the oocyte to embryo quality. Theriogenol 65 , 126-136.

Squirrell, J.M., Schramm, R.D., Paprocki, A.M., Wokosin, D.L. \& BAVISTER, B.D. (2003). Imaging mitochondrial organization in living primate oocytes and embryos using multiphoton microscopy. Microsc Microanal 9, 190-201.

Squirrell, J.M. \& White, J.G. (2004). Using multiphoton excitation to explore the murky depths of developing embryos. In Germ Cell Protocols, Volume 2: Molecular Embryo Analysis, Live Imaging, Transgenesis and Cloning, Schatten, H. (Ed.), pp. 113136. New York: Humana Press.
Squirrell, J.M., Wokosin, D.L., White, J.G. \& Bavister, B.D. (1999). Long-term two-photon fluorescence imaging of mammalian embryos without compromising viability. Nature Biotechnol 17, 763-767.

Tanghe, S., Van Soom, A., Mehrzad, J., Maes, D., Duchateau, L. \& De Kruif, A. (2003). Cumulus contributions during bovine fertilization in vitro. Theriogenol 60, 135-149.

Vajta, G., Peura, T.T., Holm, P., Paldi, K., Greve, T., Trounson, A.O. \& Callesen, H. (2000). New method for culture of zona-included or zona-free embryos: The Well of the Well (WOW) system. Mol Reprod Dev 55, 256-264.

Vejlsted, M., Avery, B., Schmidt, M., Greve, T., Alexopoulos, N. \& Maddox-Hyttel, P. (2005). Ultrastructural and immunohistochemical characterization of the bovine epiblast. Biol Reprod 72, 678-686.

Willadsen, S. (1973). Bovine æg in vitro \& in vivo. Thesis, Doctor Veterinary Science, pp. 1-124.

Xu, K.P., Greve, T., Smith, S. \& Hyttel, P. (1986). Chronological changes of bovine follicular oocyte maturation in vitro. Acta Veterinaria Scandinavia 27, 505-506.

Zipfel, W.R., Williams, R.M. \& WebB, W.W. (2003). Nonlinear magic: Multiphoton microscopy in the biosciences. Nat Biotech 21, 1369-1377.

Zuccotti, M., Rossi, P.G., Martinez, A., Garagna, S., ForaBosco, A. \& Redi, C.A. (1998). Meiotic and developmental competence of mouse antral oocytes. Biol Reprod 58, 700-704. 